\section{INTERMITTENT AORTIC CROSSCLAMPING PREVENTS CUMULATIVE ADENOSINE TRIPHOSPHATE DEPLETION, VENTRICULAR FIBRILLATION, AND DYSFUNCTION (STUNNING): IS IT PRECONDITIONING?}

This study was designed to determine whether intermittent warm aortic crossclamping induces cumulative myocardial stunning or if the myocardium becomes preconditioned after the first episode of ischemia in canine models in vivo. The role of adenosine triphosphate catabolism and subsequent release of purines on reperfusion-mediated postischemic ventricular dysfunction and arrhythmias was assessed with the use of selective inhibitors of nucleoside transport, p-nitrobenzylthioinosine (NBMPR), and a specific adenosine deaminase inhibitor, erythro-9-[2-hydroxy-3-nonyl] adenine (EHNA). Thirty-two anesthetized dogs were instrumented to monitor left ventricular contractility, off bypass, by sonomicrometry. During cardiopulmonary bypass dogs were treated before ischemia with either saline solution (control group, $n=8)$ or EHNA $(100 \mu \mathrm{mol} / \mathrm{L})$ and NBMPR $(25 \mu \mathrm{mol} / \mathrm{L}$ ) (EHNA/NBMPR group, $n=8$ ). Hearts were subjected to either 60 minutes of global ischemia and 120 minutes of reperfusion $(n=16)$ or 6 episodes of 10 minutes of global ischemia and 10 minutes of reperfusion, followed by 60 minutes of reperfusion $(n=16)$. Sixty minutes of sustained ischemia resulted in $\mathbf{8 0 \%}$ loss of adenosine triphosphate and induced reperfusion-mediated ventricular fibrillation and severe left ventricular dysfunction in the control group. EHNA/NBMPR treatment augmented myocardial adenosine trapping during ischemia, attenuated ventricular fibrillation, and enhanced left ventricular functional recovery, despite similar depletion of adenosine triphosphate $(80 \%$ loss $)$. In the intermittent ischemia experiment, the first episode of $\mathbf{1 0}$ minutes of ischemia and reperfusion caused significant adenosine triphosphate depletion, ventricular fibrillation, and left ventricular stunning in both control and drugtreated groups. The prevalence of ventricular fibrillation was greater in the control group than in the drug-treated group after the first episode of ischemia $(p<0.05)$. Adenosine was the major nucleoside accumulated in the myocardium at the end of 10 minutes of ischemia in the EHNA/ NBMPR-treated group ( $p<0.05$ versus control). Subsequent episodes of ischemia prevented ventricular fibrillation and did not cause cumulative left ventricular stunning in either group. Left ventricular function fully recovered in the EHNA/NBMPR-treated group after intermittent ischemia, but remained stunned in the control group. Unlike sustained ischemia, intermittent ischemia and reperfusion preserved myocardial adenosine triphosphate, limited purine release, and prevented ventricular fibrillation and cumulative stunning. These results suggest that intermittent ischemia and reperfusion augmented the endogenous protective

Anwar S. Abd-Elfattah, PhD, Mai Ding, MD, and Andrew S. Wechsler, MD, Richmond, $\mathrm{Va}$.
From the Cardiovascular Research Laboratories, Division of Cardiothoracic Surgery, Department of Surgery, Medical College of Virginia, Virginia Commonwealth University, Richmond, Va.

Supported in part by an AHA-VA grant-in-aid 90-G-44 and NIH grant HL-5-1090 (ASA).

Received for publication April 28, 1994.

Accepted for publication Dec. 20, 1994.
Address for reprints: Anwar S. Abd-Elfattah, PhD, Department of Surgery, Medical College of Virginia, P.O. Box 980532, Richmond, VA 23298-0532.

J Thorac CARDIOvaSC SURg 1995;110:328-39

Copyright (C) 1995 by Mosby-Year Book, Inc.

$0022-5223 / 95 \$ 3.00+0 \quad \mathbf{1 2 / 1 / 6 3 0 1 5}$ 


\begin{abstract}
mechanism or mechanisms of "preconditioning." Nucleoside trapping improved functional recovery after sustained or repetitive ischemia. It is concluded that adenosine triphosphate preservation or blockade of nucleoside transport may play an important role in the activation of endogenous myocardial protective mechanisms that "precondition" against subsequent ischemic stress. (J THORAC CARDIOVASC SURG 1995;110:328-39)
\end{abstract}

A lthough hypothermic cardioplegic arrest has been the procedure of choice for myocardial protection during coronary artery bypass grafting, some surgeons continue to use an old technique of intermittent noncardioplegic warm aortic crossclamping with satisfactory results, and interest in this technique has been renewed. Mechanisms underlying myocardial injury during warm intermittent global ischernia and reperfusion are not known. ${ }^{1-3}$ Recently, intermittent warm cardioplegic arrest has been used as a mean of myocardial protection during elective cardiac operation. ${ }^{1}$

A brief coronary artery occlusion provokes depletion of adenosine triphosphate (ATP), reperfusionmediated ventricular fibrillation, and persistent ventricular dysfunction referred to as myocardial stunning. ${ }^{4-8}$ On the other hand, repetitive brief coronary artery occlusion and reperfusion have been shown to significantly reduce the rate of ATP depletion and concomitant accumulation of ATP catabolites $^{9,10}$ and to limit the infarct size after a sublethal period of sustained ischemia and reperfusion. ${ }^{11}$ A single episode of 5 minutes of coronary artery occlusion produced a similar antiinfarction response. ${ }^{12}$ These observations suggested that the myocardium becomes more tolerant to ischemia by activating an adaptive response to short periods of ischemic stress. The reduction of the infarct size after brief ischemia and reperfusion followed by prolonged ischemia and reperfusion has been referred to as ischemic preconditioning. ${ }^{11,12}$ It is not known whether a brief episode of ischemia and reperfusion also limits ventricular dysfunction after intermittent ischemia and reperfusion in a canine model of warm aortic crossclamping.

The mechanisms of myocardial stunning and ischemic preconditioning have not been fully elucidated because of age-, species-, and model-related differences. $^{13}$ Oxygen-derived free radicals ${ }^{8,14}$ and impairment of contractile apparatus and handling of calcium by sarcoplasmic reticulum ${ }^{15}$ have been implicated in nnyocardial stunning. Preservation of myocardial ATP, ${ }^{11}$ activation of $A_{1}$ receptors, ${ }^{12}$ and ATP-sensitive potassium channels ${ }^{16}$ and induction of heat-shock proteins ${ }^{17}$ are among potential mechanisms of cardioprotection associated with ischemic preconditioning. Pharmacologic trapping of endogenous adenosine by a selective nucleoside transport blocker, p-nitrobenzylthioinosine (NBMPR), and a specific adenosine deaminase inhibitor, erythro-9[hydroxy-3-nonyl]adenine (EHNA), has been shown to attenuate ventricular dysfunction after 30 and 60 minutes of ischemia in $\operatorname{dogs}^{18-21}$ without a prior ischemic preconditioning episode. It is not known whether entrapment of endogenous adenine nucleoside augments ventricular functional recovery and attenuates ventricular arrhythmias during intermittent aortic crossclamping and reperfusion.

In the clinical setting, myocardial stunning and ischemic preconditioning may occur during repeated angina, angioplasty, or thrombolysis. ${ }^{22-24}$ Whether the phenomena of myocardial stunning and preconditioning occur during cardiac operation with noncardioplegic warm intermittent aortic crossclamping is not known. The role of ATP depletion and nucleoside accumulation and release during intermittent ischemia and reperfusion was investigated in the present studies.

\section{Material and methods}

The following study protocols conform with the guiding principles of the American Physiological Society. All animals were treated humanely in accordance with the United States Public Health Service Standards as outlined in "Principles of Laboratory Animal Care" formulated by the National Society of Medical Research and the "Guide for the Care and Use of Laboratory Animals" prepared by the National Academy of Sciences and published by the National Institutes of Health.

Material. Biochemical reagents were obtained from Sigma Chemical Company (St. Louis, Mo.). NBMPR was obtained from Aldrich Chemical Company (Milwaukee, Wis.); EHNA was purchased from Sigma Chemical Company.

Animal preparation. Thirty-two microfilaria-free dogs of either sex, weighing 19 to $26 \mathrm{~kg}$, were anesthetized with intravenous injections of sodium pentobarbital (Nembu- 
tal, $30 \mathrm{mg} / \mathrm{kg}$, Abbott Laboratories, Chicago, Ill.). Dogs were intubated and the lungs mechanically ventilated with a Bennett MA1 respirator (Puritan, Berkeley, Calif.). The azygos vein was ligated and the right phrenic nerve was transected to eliminate diaphragmatic contractions. The sinoatrial node was crushed, and the heart was paced at 150 beats/min with a Medtronic $5880 \mathrm{~A}$ pacemaker (Medtronic, Inc., Minneapolis, Minn.). Porcine-based heparin (400 units/kg, given intravenously) was injected before bypass followed by 200 units/kg per hour (ElkinsSinn Inc., Cherry Hill, N.J.). Cardiopulmonary bypass was initiated by subclavian artery cannulation for blood inflow and right atrial cannulation with use of a double-stage cannula for venous drainage. A bubble oxygenator (Cobe CML50, 100, Cobe, Lakewood, Colo.) primed with noncrossmatched homologous blood was used. Arterial blood gas values, $\mathrm{pH}$, hematocrit, and electrolyte levels were determined before and during bypass. Mean arterial perfusion pressure was maintained at $65 \mathrm{~mm} \mathrm{Hg}$ during cardiopulmonary bypass.

Assessment of left ventricular performance and reperfusion-mediated arrhythmias. Intraventricular and carotid artery micromanometer-tipped catheters (Millar Instruments, Inc., Houston, Tex.) were used to monitor left ventricular and systemic pressure, respectively. LV dimensions were obtained with pulse transit sonomicrometry (Triton Technology, San Diego, Calif.). One pair of $2.5 \mathrm{~mm}$ diameter LTZ-piezoelectric hemispheric crystals (Channel Industries, Santa Barbara, Calif.) was sewn onto the anterior and posterior myocardial wall along the minor axis of the left ventricle. The epicardial diameter of the left ventricle ranged from 40 to $66 \mathrm{~mm}$. Analog data were digitized at $200 \mathrm{~Hz}$ and stored on the hard disk of a PC 386 microcomputer. Subsequent analysis was done with the use of interactive software Crunch program developed in our laboratory. To assess left ventricular performance, the dog was first temporarily weaned from bypass. Then the left ventricular and arterial pressures were elevated to 100 to $120 \mathrm{~mm} \mathrm{Hg}$ by crossclamping the venous drainage and increasing the blood inflow through the subclavian cannula by the roller pump. The relationship of stroke work/end-diastolic length was established over a range of end-diastolic volumes produced by emptying venous blood into the cardiopulmonary bypass reservoir during 25 to 50 beats ( 10 to 20 seconds). Cardiopulmonary bypass was then reestablished. During data collection, all hemodynamic parameters changed, corresponding to the left ventriclar emptying maneuver, except the heart rate, which was kept constant by electric pacing (150 beats/min). Work loops were produced on a screen and recorded, and the relationship between stroke work and end-diastolic length was calculated using Crunch program analysis. The slope of this linear relationship is the most sensitive index of contractility, independent of the load within the physiologic range. ${ }^{25,26}$ Assessment of left ventricular function by this method is more accurate than hemodynamic and load-dependent indices of contractility such as left ventricular arterial pressure and positive and negative values of the rate of left ventricular pressure rise. The latter parameters decreased as the work loop diminished during data collection because of varying preload. The numbers of occurrences of ventricular fibril- lation and successful resuscitation were recorded and the frequency of reperfusion-mediated ventricular tachycardia was determined during 10-minute periods of intermittent or sustained reperfusion.

Assessment of adenine nucleotide pool intermediate metabolism. In a random fashion, transmural Tru-Cut needle (Travenol Laboratories, Inc., Deerfield, Ill.) biopsy specimens $(5$ to $10 \mathrm{mg}$ ) were obtained during 60 minutes of intermittent normothermic global ischemia and 60 minutes of sustained reperfusion. Biopsy samples were immediately frozen and stored in liquid nitrogen. Purines in each biopsy specimen were extracted with $12 \%$ trichloroacetic acid $\left(4^{\circ} \mathrm{C}\right)$ and neutralized. Denatured protein content from each biopsy specimen was determined as previously described by Lowry. ${ }^{27}$ Levels of myocardial ATP, adenosine diphosphate (ADP), adenosine monophosphate (AMP), adenosine, inosine, hypoxanthine, xanthine, and the oxidized form of nicotinamide-adenine dinucleotide were determined with high-performance liquid chromatography and expressed as nanomoles per milligram protein. ${ }^{28,29}$

Experimental protocol. Dogs underwent either $60 \mathrm{~min}$ utes of sustained myocardial ischemia and 120 minutes of reperfusion or six episodes of 10 minutes of normothermic global myocardial ischemia and 10 minutes of reperfusion, followed by 60 minutes of continuous perfusion during cardiopulmonary bypass. All hearts underwent 60 minutes of global warm ischemia and $\mathbf{1 2 0}$ minutes of reperfusion. To determine the role of purine release on ventricular dysfunction, arrhythmias, and adenine nucleotide pool metabolism, a selective nucleoside transport blocker, NBMPR, and an adenosine deaminase inhibitor, EHNA, were infused into the bypass reservoir before ischemia. One bolus $(500 \mathrm{ml}$ ) of either saline solution (in the control group) or a solution of EHNA $(100 \mu \mathrm{mol} / \mathrm{L})$ and NBMPR (25 $\mu \mathrm{mol} / \mathrm{L}$ ) (in the EHNA/NBMPR-treated group) was infused into the pump reservoir before ischemia. The effects of the vehicle or drugs on left ventricular contractility and the levels of myocardial adenine nucleotide and nucleoside metabolism were determined in normal hearts before ischemia. A second bolus $(500 \mathrm{ml})$ was infused immediately before sustained ischemia or before the first episode of ischemia. A third bolus $(500 \mathrm{ml})$ was infused at the beginning of 60 minutes of sustained reperfusion to ensure availability of effective inhibitor concentrations.

Transmural biopsy specimens were obtained before ischemia, at the end of each episode of ischemia and reperfusion, and during sustained reperfusion. Left ventricular performance was assessed, after the dog was weaned from bypass, from the slope of the relationship between stroke work and end-diastolic length. Postischemic reperfusion-mediated ventricular arrhythmias were documented after each ischemic episode.

Statistical analysis. Data are presented as mean plus or minus the standard error of the mean. Sequential measurements were compared with repeated-measures analysis of variance (ANOVA) with SAS software (Statistical Analysis System Institute, Cary, N.C.). Subsequent comparisons were made between groups at each individual period of the experiment by the method of Newman and Keuls. Differences were considered significant if the 

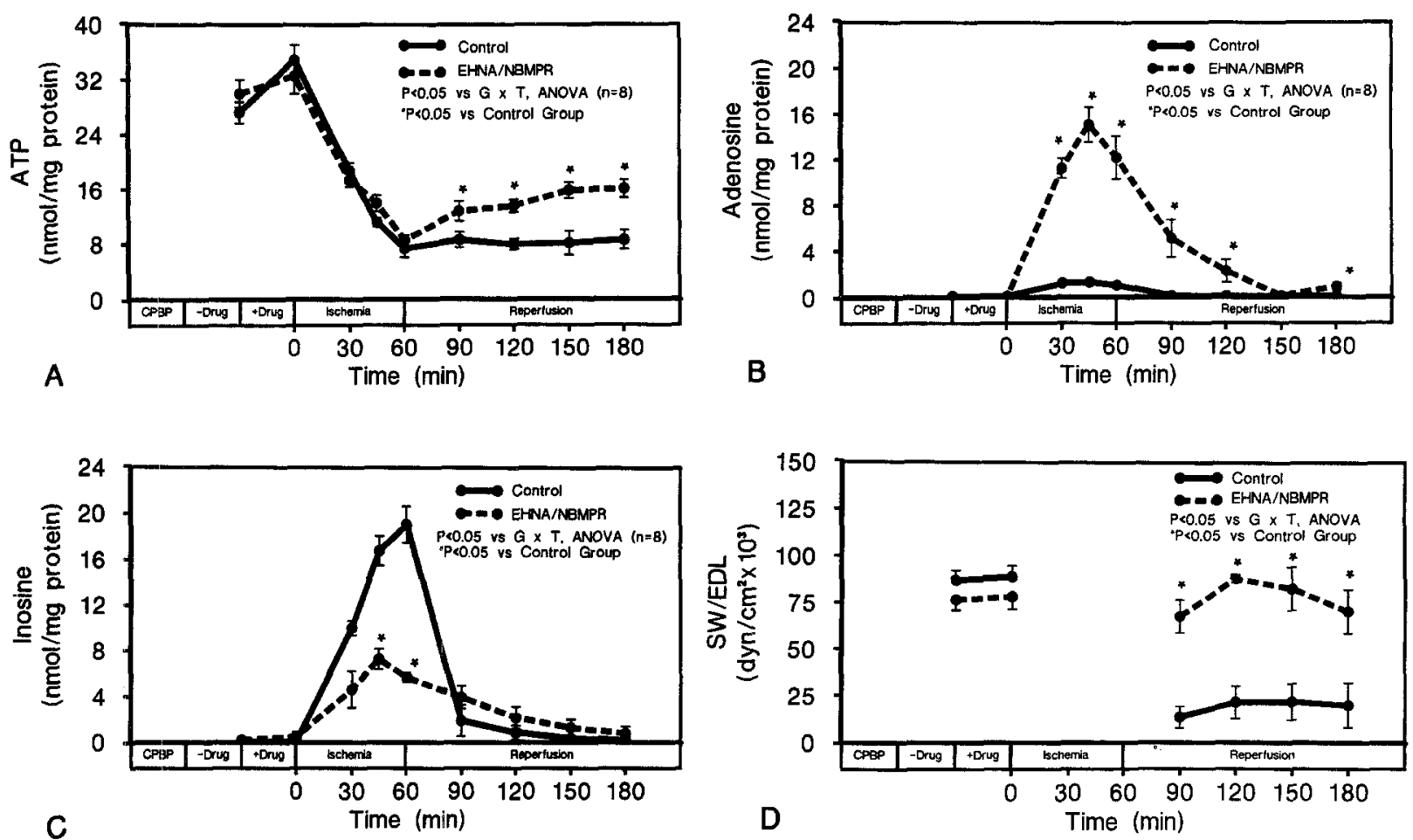

Fig. 1. Effect of sustained global ischemia on ATP metabolism and ventricular performance with and without nucleoside trapping. Effect of sustained warm ischemia ( 60 minutes) on ATP, adenosine inosine levels, and ventricular performance in presence and absence of EHNA/NBMPR. A, Effect of sustained ischemia on ATP. Levels of ATP were determined before, during, and after 60 minutes of ischemia $\left(37^{\circ} \mathrm{C}\right)$. There were statistically significant differences between groups ( $p<0.05$, ANOVA, $n=8$ each group). Significant differences between groups at each point are represented by asterisks. B, Effect of sustained ischemia on adenosine. Adenosine levels were determined before, during, and after 60 minutes of ischemia $\left(37^{\circ} \mathrm{C}\right)$. There were statistically significant differences between groups $(p<0.05$, ANOVA, $n=8$ each group). Significant differences between groups at each point are represented by asterisks. C, Effect of sustained ischemia on inosine. Inosine levels were determined before, during, and after 60 minutes of ischemia $\left(37^{\circ} \mathrm{C}\right)$. There were statistically significant differences between groups $(p<0.05$, ANOVA, $n=$ 8 each group). Significant differences between groups at each point are represented by asterisks. $\mathbf{D}$, Effect of sustained ischemia on left ventricular performance. Left ventricular performance was determined before and after vehicle of EHNA/NBMPR administration to determine the effect of drugs on normal myocardial function. There were no significant differences between groups before ischemia. Significant recovery of ventricular function after 60 minutes of sustained ischemia was observed in EHNA/NBMPR-treated group, whereas recovery was severely impaired in control group ( $p<0.05$, ANOVA, $n=8$ each group). Significant differences between groups at each point are represented by asterisks. CPBP, Cardiopulmonary bypass; $G \times T$, group and time; $S W / E D L$, stroke work/end-diastolic length.

probability value $(p)$ for comparison of best least-squares means was less than 0.05 .

\section{Results}

Sustained ischemia. Sixty minutes of sustained ischemia caused severe depletion of myocardial ATP (about 80\% loss) (Fig. 1, $A$ ) and concomitant accumulation of nucleosides (Fig. $1, B$ and $C$ ) in the control and EHNA/NBMPR-treated groups.
Frequency of reperfusion-mediated ventricular fibrillation was greater $(100 \%)$ in the control group than in the drug-treated group $(68 \%)$ after 60 minutes of sustained ischemia $(p<0.05)$. Left ventricular function was completely recovered in the EHNA/NBMPR-treated group, whereas poor recovery of function ( $23 \%$ of baseline value) was observed in the control group (Fig. $1, D)(p<$ $0.05)$. 


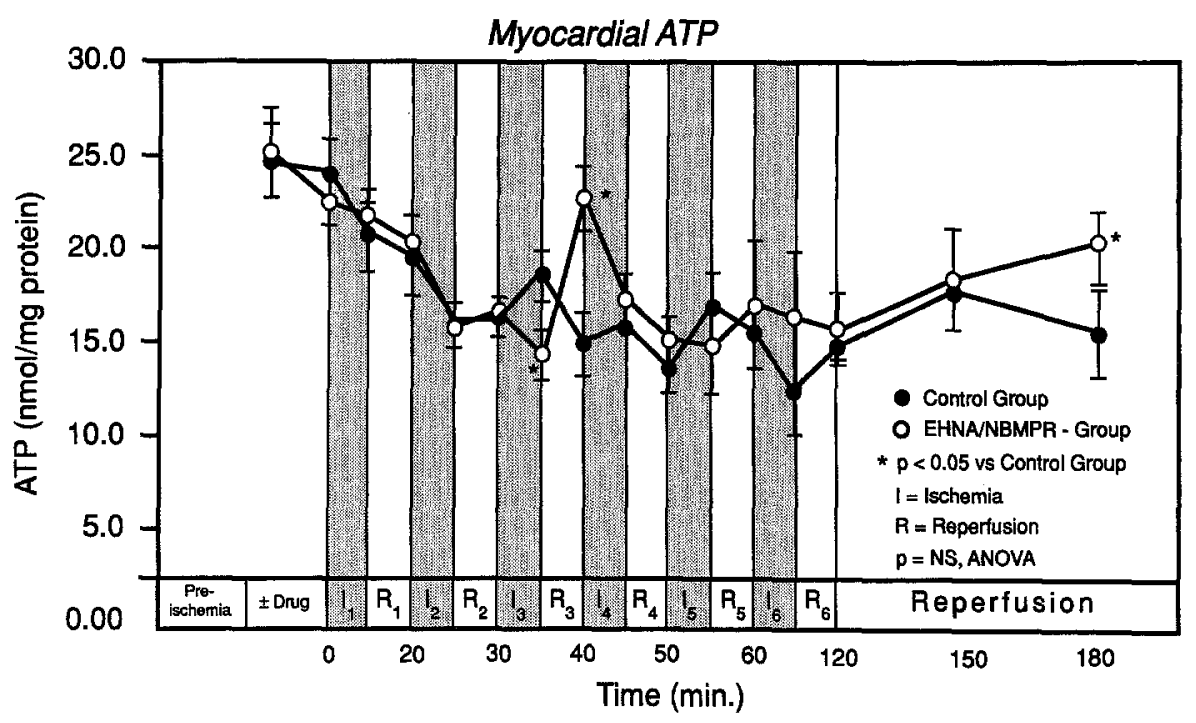

Fig. 2. Effect of intermittent ischemia on ATP with and without nucleoside trapping. Effect of intermittent global normothermic ischemia and reperfusion on myocardial ATP in presence and absence of EHNA/ NBMPR is depicted. There were no significant differences between groups with respect to ATP levels as demonstrated by ANOVA ( $p=\mathrm{NS}, n=8$ each group). However, at one point only there was significant increase in ATP between groups, as represented by asterisk.

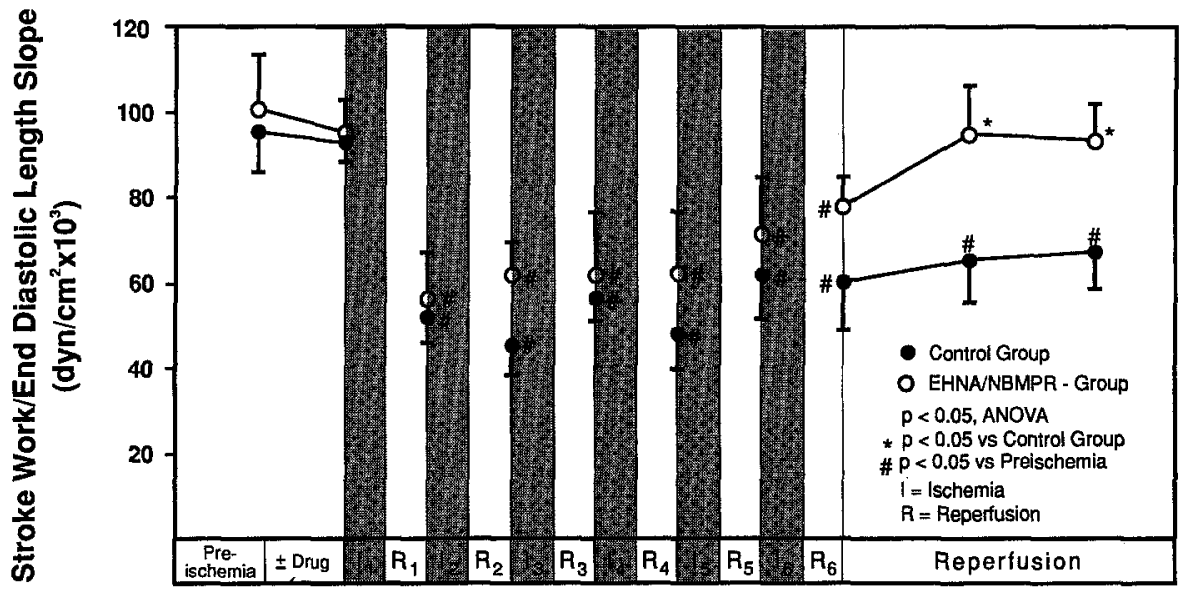

Fig. 3. Effect of intermittent ischemia on ventricular performance with and without nucleoside trapping. Effect of intermittent global normothermic ischemia and reperfusion on left ventricular function in presence and absence of EHNA/NBMPR is depicted. There were significant differences between groups after reperfusion ( $p<0.05$, ANOVA, $n=8$ each group). Significant differences between groups at each point are represented by asterisks.

Intermittent ischemia. Fig. 2 depicts the effect of intermittent normothermic global ischemia and reperfusion on ATP depletion and left ventricular performance. Administration of EHNA/NBMPR did not alter physiologic levels of adenine nucleotides (ATP, ADP, AMP), nucleosides (adenosine, inosine), or oxypurines (hypoxanthine, xanthine) before ischemia. The first 10 minutes of normothermic global ischemia resulted in slight depletion of myocardial ATP from $24.67 \pm 2.0$ to $20.59 \pm 1.8$ $\mathrm{nmol} / \mathrm{mg}$ protein in the control group $(16.54 \%$ reduction) and from $25.16 \pm 2.5$ to $21.73 \pm 1.4$ $\mathrm{nmol} / \mathrm{mg}$ protein in the drug-treated group $(13.63 \%$ reduction) (Fig. 3). Myocardial ATP levels de- 
Volume 110, Number 2

Table I. Effect of intermittent ischemia and reperfusion on myocardial adenosine

\begin{tabular}{|c|c|c|}
\hline \multirow[b]{2}{*}{ Time } & \multicolumn{2}{|c|}{ Adenosine (nmol/mg protein) } \\
\hline & Control $^{*}$ & $E H N A / N B M P R^{*}$ \\
\hline Preischemia & 0 & $0.15 \pm 0.12$ \\
\hline Vehicle or drug & $0.16 \pm 0.08$ & $0.10 \pm 0.12$ \\
\hline First ischemia & $0.38 \pm 0.04 \uparrow$ & $5.23 \pm 0.68+\div$ \\
\hline First reperfusion & $0.02 \pm 0.01 \dagger$ & $2.80 \pm 0.27+\div$ \\
\hline Second ischemia & 0 & $4.84 \pm 1.17 \dagger \ddagger$ \\
\hline Second reperfusion & $0.01 \pm 0.01$ & $0.59 \pm 0.10 \dagger+$ \\
\hline Third ischemia & $0.29 \pm 0.01 \dagger$ & $1.96 \pm 0.17+\div$ \\
\hline Third reperfusion & $0.05 \pm 0.04$ & $1.79 \pm 0.75+\div$ \\
\hline Fourth ischemia & $0.21 \pm 0.01 \dagger$ & $2.18 \pm 1.00+t$ \\
\hline Fourth reperfusion & $0.08 \pm 0.04 \uparrow$ & $1.14 \pm 0.66+t$ \\
\hline Fifth ischemia & $0.53 \pm 0.10 \dagger$ & $0.70 \pm 0.19+\div$ \\
\hline Fifth reperfusion & $0.11 \pm 0.03 \dagger$ & $0.63 \pm 0.30+\div$ \\
\hline Sixth ischemia & $0.10 \pm 0.06$ & $2.08 \pm 0.60+t$ \\
\hline Sixth reperfusion & $0.05 \pm 0.04$ & $0.22 \pm 0.18 \dagger$ \\
\hline 90 min reperfusion & $0.14 \pm 0.10$ & $0.27 \pm 0.10 \dagger$ \\
\hline 120 min reperfusion & 0 & $0.26 \pm 0.10 \dagger$ \\
\hline
\end{tabular}

creased to $15.86 \pm 1.2 \mathrm{nmol} / \mathrm{mg}$ protein $(35.7 \%$ reduction) in the control group and to $15.78 \pm 0.6 \mathrm{nmol} / \mathrm{mg}$ protein (37.28\% reduction) in the EHNA/NBMPR group after the second episode of ischemia $(p<0.05$ versus preischemia). No significant recovery of myocardial ATP values was observed within 10 minutes of reperfusion in either group, except after the third reperfusion period. Statistical analysis revealed that both groups behaved similarly with respect to the time of the experiment ( $p=\mathrm{NS}, *$ ANOVA). No further decline in myocardial ATP values was noted in either group after the third episode of ischemia and reperfusion or after 60 minutes of sustained reperfusion. A slight increase in myocardial ATP values was observed at the end of 60 minutes of reperfusion in the drugtreated group.

A transient increase in levels of myocardial ADP and AMP was observed after the first episode of ischemia, and these levels decreased during the first episode of reperfusion ( $p=$ NS between groups). Total adenine nucleotide levels followed the pattern of ATP depletion. The adenine nucleotide pool declined rapidly after the first episode of ischemia and then stabilized without further loss after the third episode of ischemia and reperfusion.

Administration of EHNA/NBMPR did not elevate the levels of adenosine or inosine in normal

$* \mathrm{NS}=$ Not significant.
Table II. Effect of intermittent ischemia and reperfusion on myocardial inosine

\begin{tabular}{lcc} 
& \multicolumn{2}{c}{ Inosine (nmol/mg protein) } \\
\cline { 2 - 3 } \multicolumn{1}{c}{ Time } & Control & EHNA/NBMPR* \\
\hline Preischemia & 0 & $0.29 \pm 0.19$ \\
Vehicle or drug & 0 & 0 \\
First ischemia & $1.60 \pm 0.20 \dagger$ & $0.36 \pm 0.30 \ddagger$ \\
First reperfusion & 0 & 0 \\
Second ischemia & $0.22 \pm 0.19 \dagger$ & $1.03 \pm 0.60 \dagger \ddagger$ \\
Second reperfusion & 0 & $0.14 \pm 0.08 \ddagger$ \\
Third ischemia & $0.33 \pm 0.14 \dagger$ & $0.12 \pm 0.06 \ddagger$ \\
Third reperfusion & 0 & 0 \\
Fourth ischemia & $0.09 \pm 0.08 \dagger$ & $0.44 \pm 0.02 \ddagger$ \\
Fourth reperfusion & 0 & $0.05 \pm 0.04$ \\
Fifth ischemia & $0.53 \pm 0.10 \dagger$ & $0.46 \pm 0.36$ \\
Fifth reperfusion & 0 & 0 \\
Sixth ischemia & $0.8 \pm 0.06$ & $0.55 \pm 0.40 \dagger$ \\
Sixth reperfusion & 0 & $0.19 \pm 0.17 \dagger$ \\
90 min reperfusion & $0.09 \pm 0.07$ & $0.07 \pm 0.04 \dagger$ \\
120 min reperfusion & 0 & 0 \\
\hline$* p<0.05$ between groups with repeated-measures ANOVA, $n=8$ each \\
group. \\
$\dagger p<0.05$ versus preischemia.
\end{tabular}

myocardium before ischemia (Tables I and II). In the intermittent ischemia experiments, adenosine levels increased during 10 minutes of warm ischemia in the EHNA/NBMPR group from 0 to $5.23 \pm 0.68$ $\mathrm{nmol} / \mathrm{mg}$ protein. Adenosine levels were significantly lower in the control group $(0.31 \pm 0.1$ $\mathrm{nmol} / \mathrm{mg}$ protein) than those in the drug-treated group $(p<0.05)$. After the first episode of reperfusion, adenosine was not detectable in the control group, whereas significant levels of adenosine ( $2.8 \pm$ $0.27 \mathrm{nmol} / \mathrm{mg}$ protein) were detected in the drugtreated group. At the end of the first 10 minutes of ischemia, inosine accumulated in the untreated control group $(1.60 \pm 0.2 \mathrm{nmol} / \mathrm{mg}$ protein $)(p<0.05$ versus preischemia) and washed out during reperfusion (Table II). Inosine levels were $1.03 \pm 0.6$ $\mathrm{nmol} / \mathrm{mg}$ protein in the drug-treated group at the end of the second episode of ischemia. During the second and third episodes of ischemia, accumulation of inosine and adenosine decreased in the control and EHNA/NBMPR groups, respectively. There was no further production of inosine in the control group after the second episode of ischemia. On the other hand, diminishing levels of adenosine were noted in the drug-treated group with subsequent ischemic and reperfusion episodes.

Before ischemia, myocardial hypoxanthine and xanthine were undetectable. However, a transient rise in myocardial hypoxanthine and xanthine levels 
was observed in both groups during the first episode of ischemia and these washed out during reperfusion. The levels of oxypurines, generated during ischemia, compared with the amounts of adenosine and inosine, were negligible. There was no significant loss of myocardial oxidized nicotinamide-adenine dinucleotide observed during warm intermittent global ischemia and reperfusion as compared with preischemic levels.

In the control group, 10 minutes of ischemia resulted in significant ventricular dysfunction (myocardial stunning) in both groups (Fig. 3). The slope of stroke work/end-diastolic length recovered only to $65 \%$ of normal in both groups $(p<0.05$ versus preischemia). Subsequent episodes of ischemia and reperfusion did not cause cumulative ventricular dysfunction in the control group or in the drugtreated group. During sustained reperfusion after six episodes of ischemia and reperfusion, left ventricular function was not improved or worsened in the control group after the first episode of ischemia. Administration of EHNA/NBMPR did not change the slope of stroke work/end-diastolic length in normal hearts before ischemia. Five subsequent episodes of ischemia and reperfusion also did not cause additional stunning over that induced by the first ischemic episode in the drug-treated group. There were no significant differences between groups, after each episode of intermittent ischemia and reperfusion, with respect to global ventricular function $(p=\mathrm{NS})$. However, a marked recovery of left ventricular performance $\left(95.1 \pm 11.8 \mathrm{dynes} / \mathrm{cm}^{2}\right.$ $\left.\times 10^{3}\right)$ was observed in the EHNA/NBMPR-treated group during sustained reperfusion $(p<0.05$ versus control group, $p=\mathrm{NS}$ versus preischemia). Full recovery of left ventricular function was observed at 30 and 60 minutes of sustained reperfusion in the drug-treated group ( $p<0.05$ versus control group, $p=$ NS versus preischemic function).

The prevalence of reperfusion-mediated ventricular fibrillation observed after the first episode of ischemia was more pronounced in the control group than in the drug-treated group. In the control group, seven out of eight dogs had reperfusion-mediated ventricular fibrillation $(87.4 \%$ ), whereas three out of eight dog hearts fibrillated in the drug-treated group $(37.4 \%)(p<0.05)$. Frequency of ventricular tachycardia was also greater $(51 \%)$ in the control group than in the EHNA/NBMPR-treated group (13\%) $(p<0.05)$. Ventricular arrhythmias did not recur during subsequent episodes of ischemia and reperfusion in either group.

\section{Discussion}

The rationale of the present study was to determine whether intermittent warm aortic crossclamping induces cumulative myocardial stunning or whether the myocardium becomes preconditioned after the first episode of ischemia. To achieve this goal, left ventricular performance was correlated with the myocardial level of adenine nucleotides pool intermediates before and after intermittent or sustained myocardial ischemia and reperfusion in a dog model in vivo. The role of ATP catabolism and subsequent release of purines on reperfusion-mediated postischemic ventricular dysfunction and arrhythmias was assessed with the use of selective inhibitors of nucleoside transport (NBMPR) and adenosine deaminase (EHNA).

In the present studies, 60 minutes of sustained warm ischemia and reperfusion resulted in severe loss of ATP (80\%), reperfusion-mediated ventricular fibrillation, and severe ventricular dysfunction in the untreated control animals. In the EHNA/ NBMPR-treated group, a marked recovery of ventricular function was observed despite significant depletion of ATP (80\%) and concomitant accumulation of adenosine. In the intermittent ischemia model, the first episode of brief ischemia resulted in ATP depletion, ventricular fibrillation, and ventricular dysfunction (stunning) in both the control and drug-treated groups. Five additional episodes of ischemia did not cause cumulative ATP depletion, ventricular fibrillation, or stunning.

This is an important observation because of its clinical ramifications. In the clinical setting of cardiac operation, warm intermittent aortic crossclamping was used for coronary artery bypass grafting before the introduction of hypothermia and cardioplegia. This technique is still being used by a limited number of surgeons with satisfactory morbidity and mortality results. Patients undergoing intermittent warm aortic crossclamping may have myocardial stunning after the first ischemic episode and, in the meantime, this may induce an adaptive response to the first episode of oxidative stress similar to that seen in the present study.

Nucleoside trapping by EHNA/NBMPR improved ventricular functional recovery during sustained reperfusion periods in models of both sustained and intermittent ischemia. Left ventricular function remained depressed (stunned) to the same degree as that induced by the first episode of ischemia after 60 minutes of reperfusion in the control group. These results suggest that the 
stunned heart is able to recover within 30 minutes of sustained reperfusion when pretreated with EHNA/ NBMPR. Because functional recovery was measured at 30-minute intervals during reperfusion after 60 minutes of sustained ischemia, it is not known whether left ventricular function was fully recovered after 10 minutes of reperfusion. Previous studies demonstrated that pharmacologic intervention with EHNA/NBMPR before or after ischemia selectively targets postischemic reperfusion injury without attenuating metabolic derangement during ischemia. ${ }^{18-21}$

Although all animals in the present studies were subjected to 60 minutes of cumulative ischemic time, the pathophysiologic processes in the two experimental protocols might not necessarily be comparable. However, it is interesting to note that significant attenuation of cumulative ventricular dysfunction and arrhythmias occurred during intermittent aortic crossclamping in the presence or absence of inhibitors of nucleoside transport and adenosine deaminase. The mechanisms by which the heart can tolerate intermittent ischemia and reperfusion, after the first ischemic episode, are not known. However, it is well established that myocardial injury sustained by the heart during postischemic reperfusion is directly related to the duration of ischemia and myocardial salvage on reperfusion. Therefore the element of reperfusion may explain the lack of cumulative ATP derangement and ventricular dysfunction in the present study. Another possible explanation is that the first ischemic episode could have preconditioned the myocardium against additional episodes of ischemia and reperfusion in a manner similar to that previously described by limiting the infarct size. ${ }^{12,16,17}$ It is plausible that the "memory" of prior oxidative stress induced certain biochemical changes that made the heart more tolerant to five additional episodes of ischemia. Murry, Jennings, and Reimer ${ }^{11}$ showed that the rate of ATP depletion was significantly reduced during repetitive coronary artery occlusion and markedly limited the size of infarction when these hearts were further subjected to a sublethal episode of sustained coronary occlusion in dogs. In the latter study, ventricular function was not measured. It has been reported that activation of $A_{1}$ receptor, protein kinase $C$ and $K_{A T P}$ channels, and induction of heat shock proteins may play a role in reducing the size of myocardial infarction. $^{30-33}$ It is interesting to note that these interventions are also known to reduce the rate of ATP depletion during prolonged ischemia. Warner and associates ${ }^{34}$ hypothesized that preconditioned myocar- dium produces fewer hydrogen ions during sustained ischemia, thus protecting the heart against further injury.

Results from the present studies demonstrate that the ATP pool is better preserved in the model of intermittent ischemia and reperfusion than in the model of sustained ischemia. The latter adaptive response is known to be species independent. Whether this adaptive response to the first episode of ischemia in this model of intermittent global ischemia and reperfusion, that is, lack of cumulative ventricular stunning, could be referred to as a form of preconditioning, is debatable. Nucleoside trapping, during 30 minutes of a sustained ischemic period in dogs, resulted in complete recovery of ventricular function and repletion of ATP. ${ }^{18}$ These results further support previous observations that the release of ATP catabolites, but not ATP levels, plays a crucial role in the phenomena of postischemic ventricular dysfunction "stunning" and ventricular arrhythmias. ${ }^{18,20}$ Van der Heide, Reimer, and Jennings ${ }^{35}$ reported that intracoronary infusion of adenosine or an adenosine receptor agonist reduces the rate of ATP depletion during lethal episodes of ischemia.

Free radicals have been implicated in myocardial stunning after coronary artery occlusion in the absence of myocardial necrosis. ${ }^{14,19,36,37}$ Previous work from this laboratory demonstrated that sitespecific blockade of nucleoside transport only at the time of reperfusion attenuated global myocardial stunning in the absence of adenosine ${ }^{21,36,38}$ These results also suggest that free radical generation via the ATP catabolism pathway plays a role in myocardial stunning. Zuoghaib and associates ${ }^{37}$ demonstrated that intracoronary infusion of EHNA/ NBMPR augmented adenosine levels during ischemia and attenuated ventricular dysfunction independent of coronary collateral and hemodynamic changes. Changes in the sensitivity of myofilaments to calcium may also contribute to contractile dysfunction. ${ }^{39}$ It is not known whether calcium-mediated ventricular dysfunction is a secondary event to free radical-mediated membrane injury.

Whether myocardial stunning is a prerequisite for ischemic preconditioning in the present study may be surmised by the lower recovery $(71 \%$ of baseline values) of preconditioned myocardium in the untreated control group compared with $23 \%$ recovery after 60 minutes of sustained ischemia. Therefore the greater recovery of cardiac function after intermittent ischemia and reperfusion in the untreated 
control group may reflect an adaptive response to the first short episode of ischemia. Although these results support previous reports that preconditioning does not attenuate myocardial stunning, ${ }^{40,41}$ lack of cumulative ventricular dysfunction in the present study may be partially explained by an ischemic preconditioning phenomenon. Although several pieces of evidence have accumulated that argue against the notion that myocardial stunning induces the phenomenon of ischemic preconditioning, the relationship between stunning and preconditioning remains controversial. ${ }^{42-46}$ The argument is based on the following observations: (1) myocardial stunning persists for hours, whereas the preconditioning protective effect lasts 1 hour or 24 hours; (2) heat shock, $\mathrm{A}_{1}$-receptor agonists, and $\mathrm{K}_{\mathrm{ATP}}$ channel openers limit the infarct size, thus mimicking ischemic preconditioning, without the need for a brief ischemic episode ${ }^{36,37}$; and (3) inotropic stimulation by catecholamine increases ventricular contractility of the stunned myocardium. ${ }^{45}$ The latter observations may imply that myocardial stunning is not a prerequisite for ischemic preconditioning. ${ }^{46} \mathrm{~A}$ temporary increase in contractility of the stunned heart induced by infusion of catecholamine, ${ }^{45}$ cAMP, or calcium may reflect an adequate contractile reserve of the stunned heart but not necessarily abolish stunning. The heart eventually returns to its stunned state after catecholamine withdrawal. Therefore, by definition, ischemic preconditioning requires a period of ischemia, whereas heat shock, $\mathrm{A}_{1}$ agonist, and $\mathrm{K}_{\mathrm{ATP}}$ channel openers may induce intrinsic mechanisms of protection similar to those of preconditioning but not mediated by ischemia. Most of these interventions are believed to preserve ATP during sustained ischemia.

It has been reported that brief episodes ( 2.5 or 5 minutes) of ischemia and reperfusion before 15 minutes of left anterior descending artery occlusion and 2 hours of reperfusion were not associated with greater recovery of regional contractility. ${ }^{40}$ Brief ischemia (5 to 10 minutes) or low-flow ischemia, before cardioplegic arrest at $34^{\circ} \mathrm{C}$, did not improve left ventricular developed pressure in isolated perfused rabbit hearts. ${ }^{41}$ Gall and colleagues ${ }^{47}$ demonstrated that although repetitive coronary artery occlusion does not result in cumulative ischemic dysfunction, prior ischemic events significantly alter recovery of postischemic reperfusion. In the latter study, repetitive regional left anterior descending artery occlusion and reperfusion delayed recovery of myocardial creep but not recovery of systolic func- tion. Bolli and associates ${ }^{48}$ demonstrated that better regional contractility, as measured by recovery of wall thickening, was associated with a few episodes of left anterior descending artery occlusion but did not continue after 10 occlusions. In the present study, lack of cumulative left ventricular dysfunction and ventricular fibrillation after brief ischemia (10 minutes) suggests that the myocardium became tolerant to additional repetitive global ischemia (preconditioning). Therefore one should not anticipate greater recovery of function or attenuation of myocardial stunning after the first episode of ischemia, but the beneficial effects of brief ischemia and reperfusion are expressed by the limitation of further deterioration of left ventricular function with additional episodes of ischemia (preconditioning).

In the present study, nucleoside trapping accelerated functional recovery of the stunned myocardium when dogs were treated with EHNA/NBMPR. This drug intervention has been shown to attenuate reperfusion-mediated injury by preventing generation of purine-mediated free radical production and subsequent vascular stunning. Failure to achieve a greater recovery of function ${ }^{40-46}$ could be related to persistent vascular damage as a result of injury sustained by the heart during brief ischemia and reperfusion. Stahl, Aversano, and Becker ${ }^{49}$ demonstrated that selective enhancement of function of the stunned myocardium was observed by increasing coronary flow. In that study, one would expect that augmentation of contractility would be abolished when the coronary flow returned to the level of that in the stunned heart. Therefore greater recovery of left ventricular performance in the EHNA/NBMPR group, in the present study, may be related to attenuation of reperfusion injury-mediated vascular injury in addition to that induced by ischemic preconditioning.

Chen and associates ${ }^{50}$ reported hemodynamic recovery after four 15-minute episodes of intermittent crossclamping in the presence or absence of lidoflazine in dogs. The mechanisms by which lidoflazine, as a nucleoside transport inhibitor, allowed continuous release of adenosine is not known. Myocardial protection by lidoflazine was attributed to endogenous adenosine cardioprotective effects. Results from the same laboratory have demonstrated failure of R75231, a more selective inhibitor than lidoflazine, to improve ventricular function, despite significant accumulation of endogenous adenosine after four 15-minute episodes of intermittent ischemia and reperfusion. ${ }^{51}$

Selective blockade of nucleoside transport by 
NBMPR and EHNA attenuated myocardial stunning after a short period of reperfusion. ${ }^{18-21,36-38}$ The mechanisms of cardioprotective action of EHNA/NBMPR are believed to be related to sitespecific entrapment of nucleosides. ATP repletion after 30 minutes of global ischemia and reperfu$\operatorname{sion}^{18}$ suggests that EHNA/NBMPR-trapped nucleosides are most likely to be compartmentalized inside cells. Indeed, Walsh and colleagues ${ }^{52}$ demonstrated that EHNA/NBMPR significantly reduced adenosine release from anoxic rabbit hearts, suggesting that adenosine is trapped in an intracellular compartment. Preservation of ATP seems to play a major role in myocardial preconditioning, as previously proposed. ${ }^{9-11,35}$ The mechanism by which ATP is spared, although crucial, is not known. Other cardioprotective mechanisms of preconditioning have been investigated, including $A_{1}$ and $A_{3}$ receptor activation, $\mathrm{K}_{\mathrm{ATP}}$ channels, and heat-shock proteins. ${ }^{15-17}$

Whether enhanced ventricular functional recovery, after documented myocardial stunning by pretreatment with EHNA/NBMPR, is related to entrapment of endogenous adenosine is not clear from the present study. However, it is well established that adenosine limits the infarct size after prolonged ischemia and reperfusion ${ }^{30}$ in a manner similar to that induced by ischemic preconditioning. Adenosine also activates $\mathrm{A}_{1}$ receptor and $\mathrm{K}_{\mathrm{ATP}}$ channels. Although infusion of exogenous adenosine can mimic ischemic preconditioning, it failed to increase myocardial tolerance in terms of improvement of left ventricular developed pressure in isolated perfused rat hearts. ${ }^{53}$ Therefore the definition of ischemic preconditioning may need to be broadened to include adaptive responses (memory) induced by prior stressful experiences such as heat shock, beta receptor stimulation, anoxia, alcohol, aspirin, and anesthesia. It is plausible that lack of cumulative stunning is evidence of myocardial preconditioning.

It is concluded that one brief episode of 10 minutes of normothermic global ischemia and reperfusion caused myocardial stunning but augmented myocardial tolerance (that is, preventing cumulative stunning) when hearts were subjected to five subsequent episodes of ischemia and reperfusion. Selective nucleoside trapping may play a crucial role in myocardial protection against reperfusion-mediated injury and arrhythmias in these models of global ischemia and reperfusion. An understanding of the mechanisms by which ATP is spared during subsequent episodes of ischemia is important and deserves further investigation.

\section{REFERENCES}

1. Lichtenstein SV, Ashe KA, EL Dalati H, Cusimano RJ, Panos A, Stutsky AS. Warm heart surgery. J ThORAC CARDIOvasc SuRg 1991;101:269-74.

2. Bonchek LI, Burlingame MW, Vazales BE, Lundy EF, Gassmann CJ. Applicability of noncardioplegic coronary bypass to high-risk patients: selection of patients, technique, and clinical experience in 3000 patients. J Thorac Cardiovasc Surg 1992;103:230-7.

3. Braimbridge $M$. Discussion of coronary bypass with substrate enhanced cardioplegia versus noncardioplegic technique for early revascularization in acute infarction. Eur J Cardiothorac Surg 1991;4:124-9.

4. Hendricks GR, Millard RW, McRitchie RJ, Maroko PR, Vatner SF. Regional myocardial functional and electrophysiological alterations after brief coronary occlusion in conscious dogs. J Clin Invest 1975;56:97885.

5. Weiner JM, Apstein CS, Arthur JH, Pirada FA, Hood WB Jr. Persistence of myocardial injury following brief periods of coronary artery occlusion. Cardiovasc Res 1976;10:678-86.

6. Brunwald E, Kloner RA. The stunned myocardium: prolonged postischemic ventricular dysfunction. Circulation 1982;66:1146-9.

7. DeBoer LWV, Ingwall JS, Kloner RA, Braunwald E. Prolonged derangement of canine myocardial purine metabolism after a brief coronary artery occlusion not associated with anatomic evidence of necrosis. Proc Natl Acad Sci U S A 1980;77:5471-4.

8. Triana JF, Li XY, Jamaluddin U, Thornby JI, Bolli R. Postischemic myocardial "stunning": identification of major differences between the open-chest and the conscious dog and evaluation of the oxygen radical hypothesis in the conscious dog. Circ Res 1991;69:731-47.

9. Swain JL, Sabina RL, Greenfield JC Jr, Holmes EW. Repetitive episodes of brief ischemia $(12 \mathrm{~min})$ do not produce a cumulative depletion of high energy phosphate compounds. Cardiovasc Res 1984;18:264-9.

10. Reimer KA, Murry CE, Yamasawa I, Hill ML, Jennings RB. Four brief episodes of myocardial ischemia cause no accumulative ATP loss or necrosis. Am J Physiol 1986;251:H1306-15.

11. Murry C, Jennings R, Reimer K. Preconditioning with ischemia: a delay in lethal cell injury in ischemic myocardium. Circulation 1986;74:1124-36.

12. Liu GS, Thornton J, Van Winkle DM, Stanley AWH, Olsson RA, Downey J. Protection against infarction afforded by preconditioning is mediated by $A_{1}$-adenosine receptors in rabbit heart. Circulation 1991;84:350-6.

13. Abd-Elfattah AS, Ding M, Wechsler AS. Myocardial stunning and preconditioning: age-, species-, and 
model-related differences in myocardial injury and protection. J Cardiac Surg 1993;5:311-7.

14. Bolli R. Oxygen-derived free radicals and myocardial reperfusion injury: an overview. Cardiovasc Drugs Ther 1991;5(suppl 2):249-68.

15. Krause SM. Effect of global myocardial stunning on $\mathrm{Ca} 2(+)$-sensitive myofibrillar ATPase activity and creatine kinase kinetics. Am J Physiol 1990;259(3 Pt 2):H813-9.

16. Gross GJ, Auchampach JA. Blockade of ATP-sensitive potassium channels prevents myocardial preconditioning in dogs. Circ Res 1992;70:223-33.

17. Yellon DM, Latchman DS. Stress proteins and myocardial protection. J Mol Cell Cardiol 1992;24:113-24.

18. Abd-Elfattah AS, Jessen ME, Lekven J, Doherty NE III, Brunsting LA, Wechsler AS. Myocardial reperfusion injury: role of myocardial hypoxanthine and xanthine on "free-radical-mediated" reperfusion injury. Circulation 1988; 78(Suppl):III224-35.

19. Abd-Elfattah AS, Jessen ME, Hanan SA, Tuchy G, Wechsler AS. Is adenosine-5'-triphosphate derangement or free-radical-mediated injury the major cause of ventricular dysfunction during reperfusion: role of adenine nucleoside transport in myocardial reperfusion injury. Circulation 1990;82(Suppl):IV341-50.

20. Abd-Elfattah AS, Wechsler AS. Protection of the stunned myocardium: selective nucleoside transport blocker administered after 20 minutes of ischemia augments recovery of ventricular function. Circulation 1993;88(Suppl):II336-43.

21. Abd-Elfattah AS, Jessen ME, Wechsler AS. Nucleoside trapping during reperfusion prevents ventricular dysfunction, "stunning," in absence of adenosine: possible separation between ischemic and reperfusion injury. J THORAC CARdiovasc SURg 1994;108:269-78.

22. Kloner RA, Allen J, Cox TA, Zheng Y, Ruiz CE. Stunned left ventricular myocardium after exercise treadmill testing in coronary artery disease. Am J Cardiol 1991;68:329-34.

23. Pfisterer M, Zuber M, Wenzel R, Burkart F. Prolonged myocardial stunning after thrombolysis: can left ventricular function be assessed definitely at hospital discharge? Eur Heart J 1991;12:214-7.

24. Renkin J, Wijns W, Ladha Z, Col J. Reversal of segmental hypokinesis by coronary angioplasty in patients with unstable angina, persistent $T$ wave inversion, and left anterior descending artery stenosis: additional evidence for myocardial stunning in humans. Circulation 1990;82:913-21.

25. Glower DD, Spratt JA, Snow ND, et al. Linearity of the Frank-Starling relationship in the intact heart: the concept of preload recruitable stroke work. Circulation 1985;71:994-1009.

26. Morris JJ III, Pellom GL, Murphy CE, Salter DR, Goldstein JG, Wechsler AS. Quantification of the contractile response: assessment of the work-length relationship in the intact heart. Circulation 1987;76: 717-27.

27. Lowry OH, Rosenbrough NJ, Farr AL, Randall RJ. Protein measurement with Folin phenol reagent. J Biol Chem 1951;193:265-75.

28. Hull-Ryde EA, Lewis WR, Veronne CD, Lowe JE. Simple step gradient elution of the major high-energy compounds and their catabolites in cardiac muscle using high performance liquid chromatography. J Chromatogr B Biomed Appl 1986;377:165-74.

29. Abd-Elfattah AS, Wechsler AS. Superiority of HPLC to assay for enzymes regulating adenine nucleotidase pool intermediates metabolism: 5 'nucleotidase, adenylate deaminase, adenosine deaminase and adenylosuccinate lyase-a simple and rapid determination of adenosine. J Liquid Chromatogr 1987;10:2653-94.

30. Thornton JD, Liu GS, Olsson RA, Downey JM. Intravenous pretreatment of $A_{1}$-selective adenosine analogues protects the heart against infarction. Circulation 1992;85:659-65.

31. Ytrehus K, Liu Y, Downey JM. Preconditioning protects the ischemic rabbit heart by protein kinase $\mathrm{C}$ activation. Am J Physiol 1994;266:H1145-52.

32. Gross GJ, Yao Z, Auchampach JA. $\mathrm{K}_{\mathrm{ATP}}$ channels, adenosine receptors and ischemic preconditioning. In: Abd-Elfattah ASA, Wechsler AS, eds. Purines and myocardial protection. Boston: Kluwer Academic Publishers. (In press).

33. Marber MS, Walker JM, Latchman DS, Yellon DM. Myocardial protection after whole body heat stress in the rabbit is dependent on metabolic substrate and is related to the amount of the inducible $70-\mathrm{KD}$ heat stress protein. J Clin Invest 1994;93:1087-94.

34. Warner KG, Khuri SF, Deupree RH, et al. Significance of transmural diminution in regional hydrogen ion production after repeated coronary artery occlusions. Circ Res 1989;64:616-29.

35. Van der Heide RS, Reimer KA, Jennings RB. Intracoronary infusion of adenosine slows the rate of ischemic myocardial metabolism in a model of total in vitro ischemia: relationship to ischemic preconditioning. Cardiovasc Res 1993;27:669-73.

36. Abd-Elfattah AS, Ding M, Dyke CM, Dignan R, Wechsler AS. Attenuation of ventricular dysfunction "stunning" following normothermic ischemia and hypothermic cardioplegic arrest by augmentation of endogenous adenine nucleosides [Abstract]. Circulation 1992;86(Suppl):I103.

37. Zuoghaib ME, Abd-Elfattah AS, Jeroudi MO, et al. Augmentation of endogenous adenosine attenuates myocardial "stunning" independently of coronary flow or hemodynamic effects. Circulation 1993;88[part 1]:2359-69.

38. Abd-Elfattah AS, Wechsler AS. Separation between ischemic and reperfusion injury by site specific entrapment of endogenous adenosine and inosine using 
NBMPR and EHNA. J Cardiac Surg 1994;9(Suppl): 387-96.

39. Gao WD, Atar D, Backx PH, Marban E. Direct measurements of $\mathrm{Ca}^{2+}$ responsiveness of the contractile proteins in intact stunned myocardium [Abstract]. Circulation 1994;90(part 4):I646.

40. Ovize M, Przyklenk K, Hale SL, Kloner RA. Preconditioning does not attenuate myocardial stunning. Circulation 1992;85:2247-54.

41. Bolling SF, Olzanski DC, Child KF, Gallagher KP. Does cardiac "preconditioning" result in enhanced postischemic functional recovery? Surg Forum 1991; 42:239-42.

42. Miura T, Goto M, Urbe K, Endoh A, Shimamoto K, Iimura $\mathrm{O}$. Does myocardial stunning contribute to infarct size limitation by ischemic preconditioning? Circulation 1991;84:2504-12.

43. Li YW, Whittaker P, Kloner RA. The transient nature of the effect of ischemic preconditioning on myocardial infarct size and ventricular arrhythmia. Am Heart J 1992;123:246-53.

44. Liu GS, Thornton J, Van Winkle DM, Stanley AW, Olsson RA, Downey JM. Protection against infarction afforded by preconditioning is mediated by $\mathrm{A}_{1}$ adenosine receptors in rabbit heart. Circulation 1991;84:350-6.

45. Ellis SG, Wynne J, Braunwald E, Henschke CI, Sandor T, Kloner RA. Response of reperfusionsalvaged, stunned myocardium to inotropic stimulation. Am Heart J 1984;107:13-9.

46. Matsuda M, Catena TG, Vander Heide RS, Jennings RB, Reimer KA. Cardiac protection by ischaemic preconditioning is not mediated by myocardial stunning. Cardiovasc Res 1993;27:585-92.

47. Gall SA Jr, Maier GW, Gayron JW, Feneley MP, Rankin JS, Glower DD. Mechanism of delayed myocardial recovery after repetitive ischemic episodes. Surg Forum 1991;42:243-5.

48. Bolli R, Zuyghaib M, Li XY, et al. Stunning after repetitive ischemia is caused by recurrent bursts of free radical production without a preconditioning effect [Abstract]. Circulation 1993;88(part 2):I130.

49. Stahl LD, Aversano TR, Becker LC. Selective enhancement of function of stunned myocardium by increased coronary flow. Circulation 1986;74:843-51.

50. Chen CC, Masuda M, Szabo Z, et al. Nucleoside transport inhibitor lidoflazine induced cardioprotection during intermittent aortic cross-clamping. J THORAC CARDIOVASC SURG 1992;104:1602-9.

51. Flameng W, Chen CC, Mubagwa K. Accumulation of endogenous adenosine by nucleoside transport inhibition failed to protect the ischemic myocardium [Abstract]. J Mol Ce1l Cardiol 1992;24(Suppl II): S14.

52. Walsh R, Abd-Elfattah AS, Daly JJF, Wechsler AS, Downey JM. Nucleoside transport inhibition prevents ischemic preconditioning [Abstract]. Circulation 1993; 88(part 2):I432.

53. Hendrikx M, Toshima Y, Mubagwa K, Flameng W. Improved functional recovery after ischemic preconditioning in the globally ischemic rabbit heart is not mediated by adenosine $\mathrm{A}_{1}$-receptor activation [Abstract]. Circulation 1992;86(Suppl):I342.

\section{1-800-55-MOSBY}

This number links you to the full text of articles published in over 25,000 journals, including all Mosby journals. MOSBY Document Express $^{\circledR}$, a rapid response information retrieval service, provides quick turnaround, 24-hour availability, and speedy delivery methods. For inquiries and pricing information, call our toll-free, 24-hour order line: 1-800-55-MOSBY; outside the United States: 415-259-5046; fax: 415-259-5019; E-mail: mosbyexp@class.org. 